\title{
Trends
}

1. Microbes express many competitive phenotypes in the presence of others: exploitative phenotypes include metabolic changes that increase growth rates or secreting molecules to harvest nutrients, while interference competition occurs through antimicrobial secretions or contact-dependent killing.

2. Microbial competition is common, although evidence suggests that in many environments, inter-species interactions are weak.

3. Competition is expected on first encounter, but can be reduced over time through competitive exclusion, niche partitioning or spatial separation, leading to communities with a reduced local diversity of strains and species that can nevertheless coexist stably.

4. Many complementary methods exist to study microbial communities. Combining them to analyse a simple community would reveal a more complete picture. 


\title{
The Ecology and Evolution of Microbial Competition
}

\author{
Melanie Ghoul ${ }^{1}$ and Sara Mitri2, ${ }^{2}$ \\ 1. Department of Zoology, University of Oxford, OX1 3PS, United Kingdom \\ 2. Department of Fundamental Microbiology, University of Lausanne, Unil-Sorge, $\mathrm{CH}-1015$, \\ Lausanne, Switzerland \\ *Correspondence: sara.mitri@unil.ch (S. Mitri).

\begin{abstract}
Microbes are typically surrounded by different strains and species with whom they compete for scarce nutrients and limited space. Given such challenging living conditions, microbes have evolved a plethora of phenotypes with which they can outcompete and displace their neighbours: secretions to harvest resources, loss of costly genes whose products can be obtained from others, stabbing and poisoning neighbouring cells, or colonising spaces while preventing others from doing so. These competitive phenotypes appear to be common, although evidence suggests that over time competition dies down locally, often leading to stable coexistence of genetically distinct lineages. Nevertheless, the selective forces acting on competition and the resulting evolutionary fates of the different players depend on ecological conditions in a way that is not yet well understood. Here, we highlight the remaining open questions and the theoretical predictions of the long-term dynamics of competition that remain to be tested. Establishing a clearer understanding of microbial competition will allow us to better predict their behaviour, and to control and manipulate microbial communities for industrial, environmental and medical purposes.
\end{abstract}

Keywords: interference competition, exploitative competition, bacteria, communities, social evolution.

\section{The Nature of Microbial Competition}

Microbes dominate the tree of life in species number and diversity (see Glossary), and inhabit the largest range of environments on earth. Like macroorganisms, microorganisms too live in a miniature entangled bank, where some species are tightly associated and rely heavily on each other to survive, such as the microbial guilds that convert nitrogen in the atmosphere to its various forms in the soil, or the symbiotic microbes that provide health benefits to their hosts. However, given the density in which microbes are found and the scarcity of resources in most environments, one cell's survival may mean starvation for another, leading to fierce competition for finite resources, be they sunlight, nutrients or space.

We consider phenotypes in a focal strain to be competitive if they cause a fitness decrease in a competitor strain, and if they are more likely to have evolved as a consequence of biotic competition rather than environmental pressures. Competitors must overlap in resource use, which excludes behaviours such as predation and parasitism that also reduce the fitness of one of the players. The competing strains that we refer to throughout the article can differ only by a single mutation or can be distantly related species.

The two main resources necessary for microbial survival are nutrients and space. Nutrients essential for growth and metabolic functions include: light, carbon, nitrogen, phosphorus, 
sulphur, hydrogen, oxygen, calcium, iron and other metals [1-4]. Resource concentrations will vary between environments, such that microbes will be in competition for the limited components. As they grow and produce more biomass, microbial groups expand in space, and compete with others to colonise areas in which nutrients are abundant. A third and less commonly considered resource is genetic material. DNA is used as a nutrient source, but it may also provide its host with beneficial traits, enhancing its ability to survive and adapt [5]. The advantage of DNA uptake is particularly salient in the acquisition of antibiotic resistance genes $[6,7]$, but since there is also the possibility of taking up harmful genes, the net consequences of DNA uptake on microbial fitness remain unclear.

\section{Competitive Phenotypes}

There are two ways in which microbes compete for the resources listed above: (i) indirectly through exploitative competition, which occurs through resource consumption (passive competition) and (ii) directly through interference competition, where individual cells damage one another (active, chemical warfare).

Exploitative competition involves the consumption of a limiting resource by one strain restricting its supply from the competitor. This occurs either through increased nutrient uptake or through the extracellular secretion of molecules that harvest nutrients. As an example of the former, both Saccharomyces cerevisiae and Escherichia coli can metabolically shift from fermentation to respiration when oxygen is present, generating high growth rates but low yield, allowing them to absorb nutrients faster than their competitors [8-10]. Examples of the latter competitive strategy include the production of digestive enzymes to degrade complex nutrient molecules, or siderophores, which are iron-scavenging molecules that access insoluble iron. However, these molecules are often costly, and because they are secreted outside of the producing cell, they are also 'public goods' that benefit neighbouring cells. Therefore, another competitive mechanism is to exploit the products secreted by others, and lose or reduce a strain's own secretions, a strategy often referred to as 'cheating'. Of the best-studied systems involving the interplay between these two competitive mechanisms - cooperation that allows more access to nutrients, and cheating that saves the cost but relies on the presence of cooperators - is the production of iron-chelating siderophores [11-15] and of quorum sensing (QS) molecules that coordinate the expression and production of exofactors [16, 17].

Strains also compete to position themselves in prime locations within a niche while preventing others from accessing it [18]. This can be achieved either by rapidly colonising uninhabited spaces or by killing or pushing out already established competitors [19]. A variety of molecules are involved in these strategies: rhamnolipids allow cells to swim to new areas or push competitors away [20, 21]; adhesins bind to surfaces and prevent displacement by invaders [22]; extracellular polysaccharides (EPS) can smother and starve competitors, while also pushing clone-mates into nutrient-rich environments [18, 23, 24] (Figure 1A). Some microbes, such as Myxobacteria xanthus and Dictyostelium discoideum produce fruiting bodies to glide toward food sources, and limit the diffusion of extracellular digestive enzymes outside of the fruiting body. In doing so, they achieve both enhanced motility to access new niches, and adhesion to closely related cells to gain biomass and keep competitors away [24, 25].

Similarly to these fruiting bodies, many microbes form cell aggregates - commonly known as biofilms - that protect cells from antimicrobials, predators and other environmental hazards. Inhibiting the formation of these biofilms in others is another competitive strategy [26]. For example, on entry into biofilm, E. coli cells produce surfactants and EPS that inhibit biofilm 
102

103

104

105

106

107

108

109

110

111

formation in Staphylococcus aureus and Pseudomonas aeruginosa [27, 28]. Similarly, P. aeruginosa cells swarm over a surface and occupy it to form a biofilm, a behavior termed 'surface blanketing', which prevents Agrobacterium tumefaciens from forming its own biofilm [20]. Although the overall cell number of the 'losing' strain is not necessarily reduced on biofilm expulsion, it may nevertheless suffer significant losses under certain conditions, for example in the presence of antibiotics [29, 30]. Analogously, QS inhibition molecules, which are widespread among bacteria, may mediate competition [29, 31-33]. For example, Bacillus subtilis produces enzymes that degrade QS molecules in Vibrio cholerae, which are subsequently unable to form biofilms [29, 31].

The classical example of interference competition is the production of antimicrobials, which range in their killing spectrum from strain-specific bacteriocins to more broad-spectrum peptides and antibiotics [35, 38] (Figure 1C). Although it has been proposed that at subinhibitory concentrations, antibiotics may be used for cooperative purposes, such as signaling [39, 40], recent data shows otherwise, maintaining the classical understanding of antibiotics as weapons $[41,42]$. Other mechanisms of contact-dependent interference competition include type VI secretion systems (T6SS), whereby cells inject syringe-like protrusions containing toxins and other molecules into neighbouring cells that then lyse [5, 34, 43, 44] (Figure 1B). The victim's DNA may also be transferred back into the attacker's cell [5]. The utility of taking up and integrating foreign DNA remains unclear, but some genes, such as those providing toxin immunity and antimicrobial resistance can allow a strain to sweep through to fixation [7, 45, 46].

Many of these competitive phenotypes can be differentially expressed within clonal populations. This variability can enhance a genotype's competitive success [47]. For example, clonal cells within a population can perform different physiological roles, and thereby contribute to a collective functionality [48, 49], such as enhanced growth in nutrient-fluctuating environments [50]. In cyanobacteria, a fraction of the population of cells fixes nitrogen into a usable form, while the rest undergo photosynthesis, together increasing group productivity $[47,51]$. Similarly, in the intestinal pathogen Salmonella enterica serovar Typhimurium, some cells remain in the host gut lumen and divide, while others invade the tissue and induce an inflammatory response in the host that kills off other bacteria [52]. It is essential to better understand the extent to which such phenotypic heterogeneity occurs, the various roles that different cells can play, and how this can shape competitive interactions (Box 1).

\section{Competition Between Microbes Is Widespread}

Given that so many competitive phenotypes have evolved (Table 1), competition must be an important part of microbial life. But how common is it? Are microbes largely living cooperatively with minimal conflict, or is it a constant battlefield of attack and counterattack? When is competition expected?

Data from a number of different ecosystems suggest that competition is prevalent. Genomic analyses show that $25 \%$ of Gram-negative bacteria have genes coding for a type VI secretion system [53], while virtually all actinomycetes dedicate $5-10 \%$ of their genomes to secondary metabolites [54], which include antibiotics and other potentially damaging molecules. However, we still need to discover the functions of these metabolites - what percentage of them is in fact aggressive - and perform similar analyses in other microbial groups. A powerful approach to assessing the extent of exploitative competition is by using sequence data to build and simulate metabolic models $[55,56]$. In one of the first studies using this approach, Freilich et al. predicted abundant competition between a collection of widely sampled bacterial species, and few instances of unidirectional positive interactions [55]. 
Co-culture studies have found similar patterns. Bacterial isolates from tree-holes, which are aquatic ecosystems found around the roots of beech trees, tend to compete with one another in co-culture [57-59]. Soil isolates also grow less well in the presence of other species or even in their filtered growth media [60-62]. Another example comes from the mouse gut. By fitting a generalised Lotka-Volterra network model to a dataset quantifying different bacterial sequences over time, Stein et al. [63] find that competitive interactions - albeit weak ones dominate the community $[63,64]$. Weak competitive interactions were also found in another microbiome study, this time in humans [65]. Other empirical data from the microbiome indicate that, in agreement with the 'habitat filtering' principle, species with similar resource requirements tend to live in similar areas of the body [65-67], which may explain local competition. Finally, experiments using mixtures of model bacterial species to study synergistic interactions must rely on evolving or engineering metabolic co-dependence between them as a means to get them to co-exist in the lab, indicating that in their natural state, these species may simply outcompete each other [68-71].

Even though the evidence for the high prevalence of competition is growing, some caveats need to be considered. First, the measured interactions may not be representative of those in the species' natural environments. For example, because co-culture experiments select for a subset of strains that are able to grow in the lab, they may be more likely to have similar metabolisms and compete with each other on first encounter. Second, genomic analyses suffer from another weakness: to what extent are the genes found in sequence data expressed? The difficulties of antibiotic discovery and biosynthesis indicate that expression levels may indeed be quite low $[72,73]$. We discuss the consequences of such experimental and analysis choices in more detail in Box 1.

Assuming that the pattern is real, however, when does competition occur? Why are some strains more aggressive than others? In Figure 2, we summarise our current understanding of the selective forces behind competition. Competition is predicted to be favoured under three conditions: (i) when coexisting strains have overlapping metabolic niches and require similar resources (Figure 2, top row), (ii) when cells of these different strains are spatially mixed on a scale where nutrients and secretions are shared (Figure 2, middle row), and (iii) when cell density is high relative to the available resources, such that they become limiting (Figure 2, bottom row) [74, 75].

There are many environmental factors determining whether these conditions are met (Figure 2, central column). For example, environments with a high nutrient complexity, containing multiple resources or niches can reduce selection for competition [60], particularly if each species is limited by a different resource (Resource Ratio Theory) [76, 77]. Similarly, the more phylogenetically similar species within a community are, the more likely they will occupy overlapping metabolic niches and compete for the same resources [78]. Accordingly, distantly related species will tend to consume different resources and co-exist with minimal - or even positive - effects on one another $[79,80]$. Even in the absence of phylogenetic similarity through common descent, metabolic overlap may occur through lateral transfer of metabolic genes [7, 45]. It can also result from a lack of environmental disturbances, such that few new strains arrive in the environment bringing in organisms with different metabolic needs [7].

Spatial mixing depends on multiple factors, including nutrient abundance [36, 81], and various mechanical aspects of the environment, such as its viscosity and the diffusivity of different molecules, and the frequency at which it is disturbed [82]. Cardinale [83] showed that a mixture of algal species could only coexist and take on complementary roles in removing nitrate from 
204

205

206

207

208

209

210

211

212

213

214

215

216

217

218

219

220

221

222

223

224

225

226

227

228

229

230

231

232

233

234

235

236

237

238

239

240

241

242

243

244

245

246

247

248

249

250

251

252

253

254 stream water if the flow environment was heterogeneous (different flow velocities). A uniform environment instead led to competitive exclusion [83]. Apart from ecological conditions, spatial mixing can also result from a co-dependence on the presence of a cooperating strain [84-87]. Despite these heuristics, however, the effects of environmental manipulations on competition are not straightforward to predict. Indeed, the same manipulation - for example increased viscosity or the frequency of environmental disturbances - may simultaneously drive selection for competition in opposite directions (Figure 2).

A recently proposed 'competition sensing' hypothesis suggests that cells may be able to detect and respond to competition [89, 90], whereby physiological stress responses induced by the presence of competitors are used to regulate competitive phenotypes. Some cells can then recognise and tune their responses depending on whether they sense competition through a lack of nutrients, or cellular damage $[89,90]$. Consistent with this, $P$. aeruginosa cells can detect antibiotics, and induce the formation of biofilms [91]. They can also detect when neighbouring $P$. aeruginosa cells are killed, and trigger a counterattack using their T6SS [92]. B. subtilis cells in biofilms are able to detect nearby Bacillus simplex biofilms and secrete lethal toxins that kill them [93]. The presence of neighbouring colonies also alters the competitive behaviour of many species of soil bacteria [37, 41,60] (Figure 1E). Depending on the identity of a neighbouring colony, a species pair can either upregulate or suppress its antibiotic production [41, 94].

\section{Consequences of Competition Over Time}

Most microbial communities studied in the lab are snapshots in time resulting from a history of interactions between individual cells and genotypes. But what are the consequences of competition over ecological and evolutionary time-scales? Two key measures are of interest when predicting the dynamics of a community: its diversity, and its stability.

Overall, competition is predicted to lead to a local reduction in diversity - where 'local' refers to the scale at which cells have fitness effects on each other - and an increase in ecological stability [64, 95]. However, this may occur in a number of different ways (Figure 3, Key Figure). Three ecologically stable outcomes of competition are well accepted (Figure 3A-C): (i) that the less competitive strains go extinct while others dominate the community [79, 96], (ii) that strains continue to coexist by occupying different metabolic niches, where each specialises on a different resource type, or (iii) that strains separate into different spatial niches or patches.

A nice set of examples of niche differentiation in resources (Figure 3B) comes from experimental evolution in the tree-hole communities mentioned above [57-59], where initially competing species diverged in their use of resources as they co-evolved. The species even evolved to use each other's waste products and increase overall productivity, suggesting that even when new niches are absent, species in the community can create and exploit alternative resources within the niche. Following niche differentiation then, competition can become neutralised through a reduction in interaction strength, potentially leading to symbiotic relationships and productive communities [58]. Co-existence of competitors through spatial separation (Figure $3 \mathrm{C}$ ) is possible in solid or semi-solid structures such as mucus, soil, the surface of a leaf or an agar surface, which consist of many spatial niches. This has been studied extensively in microbial colonies that begin from well-mixed populations containing millions of competing cells that expand outwards onto an agar surface and form clonal patches [36, 97, 98]. Although this process begins with the competitive exclusion of much of the original population, coexistence of multiple strains is possible in separate spatial areas, and has been shown in many different organisms and systems [71, 75, 99, 100] (Figure 1D). 
We outline three other possible scenarios following competition whose dynamics are currently less well established: First, strains may stably coexist in the same niche in a parasitic relationship (Figure 3D). The recent Black Queen Hypothesis suggests that in a group of species in which a public good is required, if all but one species lose the ability to produce it, the producing species must continue to produce to avoid its own extinction, even if it benefits others $[11-13,101]$. Similar equilibria have been described for cooperators and cheats of the same species [102-105], and for rock-paper-scissor dynamics, where cyclic dynamics occur between antibiotic producers, resistant cells (immune but do not attack) and sensitive cells [106, 107]. These ideas are supported by experimental evidence, for example in siderophore production in marine bacteria [13]. While such communities may be ecologically stable and remain diverse, their evolutionary stability is questionable, since producers may evolve to produce more private or less costly secretions [103], to eliminate their competitors through interference competition, or exploiters may evolve to produce something in return, leading to a cooperative exchange with the producer $[101,108]$.

Second (Figure 3E), if strains are unable to escape or avoid their competitors, they may maintain their aggressive phenotypes, increasingly ramp them up or diversify them in an arms race [82]. An arms race is an evolutionary process rather than an outcome of competition, and may eventually lead to one of the other outcomes (e.g. competitive exclusion). Otherwise, theory and experiments have shown that aggressive phenotypes and resistance to them can be maintained in a stable equilibrium in spatially structured populations $[19,35,107,109]$. The dynamics of stability and diversity, then, strongly depend on environmental conditions, and the nature of the competitive phenotypes. Phenotypes that incur a higher cost, for example, may be less readily maintained $[35,110]$. A study in soil bacteria found that there is a trade-off between two strategies: investing into efficient growth or into aggressive phenotypes such as antibiotics [111], a choice that may depend on environmental conditions (Figure 2), such as population density [112]. Soil Streptomyces indeed produce an exceptional range of antibiotics targeting many different species, which may be due to liquid flow in the soil, leading to more spatial mixing [81], or an increased probability of invasion. Another possibility is that as weaker strains get outcompeted in the soil, diversity is reduced. And because high diversity isolates competitors from each other through buffer zones $[85,113]$, novel warfare may be enhanced between the remaining strains as the buffer zones disappear.

A final scenario (Figure $3 \mathrm{~F}$ ) that has only recently been proposed, is that warfare between two strains can be neutralised by other community members, as has been found in studies on antibiotic antagonism [41, 94]. Kelsic et al. [94] have theoretically shown that this can lead to ecologically stable equilibria wherein different species neutralise all produced antibiotics, and diversity is maintained. On an evolutionary time-scale, however, one might expect these protective mechanisms to break down.

In sum, competition generally reduces diversity and increases ecological stability on a local scale, although some exceptions exist. Which of the long-term dynamics are expected as a consequence of competition on a larger scale likely depends on the selection pressures of a given environment as listed above and in Figure 2. In fact, in different areas of the same environment, selection may result in an arms race in one area, competitive exclusion in a second and a synergistic division of labour in a third [114]. Exactly how these factors would influence diversity, stability and the prevalence of competition and cooperation needs to be addressed by future research. 


\section{Concluding Remarks}

Microbes grow in challenging environments where scarce resources must be shared with many other strains and species. Under these conditions, microbes have evolved many competitive strategies, including rapid growth to take up resources, direct aggression to eliminate or displace others, or alternative metabolisms that benefit from and exploit the presence of competitors. While this may sound like a highly aggressive microbial world, evidence suggests that competition often drops over time, leading to stable equilibria involving weak interactions between strains that have either eliminated their competitors, or partitioned the available niches and space.

Decades of research are responsible for the details of this picture. Nevertheless, it remains preliminary. More effort will be needed to understand how these findings generalise. In particular, apart from the classical outcomes of competition, other evolutionary outcomes are less well understood and merit further focus (see Outstanding Questions). Microbial systems are excellent models to test such ecological and evolutionary predictions with scope for developing methods to compare microbial communities, and disentangle interactions within them. Progress toward this goal can be accelerated through increased exchange between ecologists and social evolutionary biologists, as well as researchers studying model systems and environmental samples (Box 1). Such collaboration would lead to more accurate and informed predictions on the nature of interactions in microbial communities. The ability to make such predictions can have many important implications in the management and design of microbial communities, whether to increase competition in soil communities to prevent the invasion of pathogens [82], or to decrease competition and thereby increase productivity in biofuel-producing communities [115]. A good understanding of microbial competition can result in expert microbial bioengineering.

\section{Box 1. Approaches and limitations to studying microbial competition}

Studying microbial competition involves different levels of abstraction. The daunting complexity of a microbial community can be approached from the bottom-up, by focusing on a small aspect or a subpopulation that is dissectable and understandable. In contrast, top-down approaches allow a bird's-eye-view of a community and the interactions within it, which lacks in-depth understanding, but covers as many components as possible.

A powerful top-down approach to studying community interactions is using genomic, transcriptomic and metabolomic data. A first analysis often involves constructing co-occurrence networks by calculating correlations in the abundance of species pairs $[116,117]$. These networks capture how diversity and species composition change over different community samples, but are not necessarily suited to interpreting interspecies interactions. This is because it is impossible to tell whether a negative correlation between a species pair is due to competitive exclusion or habitat filtering [65]. Interactions can instead be predicted by building metabolic models for different species, and simulating their growth under different resource compositions. This method has been widely applied, and standardised tools are becoming available $[55,56,65,78,118]$. However, only rarely are other social phenotypes taken into account, such as secondary metabolites (Table 1, [119]). Furthermore, the models are based on the presence or absence of genes, regardless of whether they are expressed in reality. This can be resolved using transcriptomics, by studying gene expression profiles in addition to screening for variation in the expression of genes in complexes that share the same promoter, e.g. 
bacteriocin production and immunity operons, which were previously thought to be equally expressed [120]. Finally, metabolomics can make more stringent links between gene expression and observed phenotypes by correlating them with cellular and secreted metabolites [121].

Bottom-up approaches include co-culturing different strain combinations in the lab, which is an intuitive and powerful technique where the effects of careful manipulations can be monitored over time. However, a number of issues are relevant for interpreting the results. Firstly, only a minority of environmental isolates will manage to grow in the laboratory, biasing towards lower metabolic diversity and higher competition (Figure 2). In particular, strains that rely on the presence of others to grow - where one would detect a positive interaction - will be excluded [122]. Secondly, species may meet in the lab that would never meet in reality, possibly triggering an aggressive response. This may be the case in experiments involving interactions between 'model' bacterial species, such as $E$. coli or $P$. aeruginosa. Finally, growth in the lab often occurs over short time-scales [96] in liquid cultures lacking spatial structure, and containing relatively high concentrations of nutrients whose composition is somewhat arbitrary and will certainly affect interactions $[55,65]$. Assuming that these problems can be weeded out, however, co-cultures generate high-resolution data, which can be used to seed models of cogrowth, such as generalised Lotka-Volterra models $[63,123]$.

Another general problem is that studies typically consider whole populations and ignore phenotypic variation between individual cells. As the technology of single-cell microbiology advances, methods for taking this diversity into account are becoming more readily available. Furthermore, the spatial organisation of strains in the original environment is typically destroyed through sampling. Two co-isolated strains that are found to compete in the lab may actually live in separate clonal patches that are millimeters away. Accordingly, sampling is likely to exaggerate both diversity and competition between strains. There is then a need for sampling methods that conserve spatial structure, such as fluorescent in situ microscopy, where one can follow the identity and gene expression of individual cells over different areas and over time. These approaches have advanced significantly in recent years [124].

Finally, theoretical approaches have been and can be extremely valuable in capturing and predicting the ecology and evolution of competitive interactions, particularly over large data-sets and large (evolutionary) time-scales, which are difficult to follow experimentally. These include the genomic models discussed above, which have so far focused on metabolomics, spatiallyexplicit computer simulations, which can predict the role of space on competition between genotypes $[23,81,84,85,101,125,126]$, and more abstract models, such as network models wherein diversity and stability can be calculated analytically $[64,95]$ or social evolution models that can make predictions on the frequencies of different traits and how selection will shape them over time [127, 109].

\section{Glossary}

Competition: consider two strains $A$ and $B$ that differ on one or more loci. Strain $A$ is a competitor of B if (a) B has a lower fitness in A's presence relative to its absence; (b) the phenotype in $A$ resulting in fitness change in $B$ occurs only in the long- or short-term presence of $B$; and (c) $A$ and $B$ require similar nutrients and space. Note that this definition is contextdependent. The competitive phenotype is not necessarily only expressed upon interaction with a competitor but can be constitutively expressed provided it is likely to be responsible for the fitness change during competitive interactions. 
Diversity: number of strains or species in a community (however they may be distinguished, e.g. OTUs at $97 \%$, or differentially labelled strains; a community also needs to be spatially delimited, e.g. a microbial colony, or strains living in the human oral tract). Ecological stability: the probability that a community will return to its previous state following a small perturbation. We use this definition broadly to include measures such as resilience (the speed at which a community returns to its previous state) and permanence (all original species are maintained in the community) [64].

Evolutionary stability: evolutionary stability refers to evolutionary stable strategies (ESS), a game-theoretic concept whereby a population maintaining that strategy cannot be invaded by any alternative strategy that is initially rare [128].

Fitness: here we use fitness as a proxy for the rate of division and survival relative to the interacting competitors' division and survival.

Habitat filtering: the habitat filtering principle predicts that phylogenetically similar species will tend to co-occur because the environment selects for species that are adapted to it.

Lotka-Volterra network: a system of differential equations that describes the population dynamics of two or more interacting groups (typically species).

Resource Ratio Theory: this theory states that a species in a community that is able to survive on the lowest abundance of a given nutrient will dominate the community if it is limiting. In the presence of two limiting nutrients, it predicts that two species may coexist, provided that each is limited by one of the nutrients.

\section{Figure Legends}

Figure 1. Competitive Phenotypes. (A) Secretions by a Pseudomonas fluorescens mutant (green), allowing it to break through and colonise the top of the colony of the wild-type strain (red) and eventually outgrow it [18]. Left: whole colony, right: zoomed in view of box in the left panel. (B) T6SSs in Vibrio cholerae (red, $m C h e r r y 2$ ) and $P$. aeruginosa (green, $g f p$ ) on cell contact leads to the lysis of $V$. cholerae cell (arrow) by $40 \mathrm{~s}, 4.5 \times 4.5 \mu \mathrm{m}$ images are shown [34]. (C) Soft agar plate with one central colony of colicin-producing E. coli, surrounded by an inhibition zone and colonies of sensitive bacteria [35]. (D) Competitive exclusion in space. A drop with a 1:1 mixture of $P$. aeruginosa cells labelled in either blue or yellow fluorescent protein is left to grow into a colony. Over time, lineages form the centre die off, while only a few clonal patches grow toward the colony edge [36]. (E) Streptomyces coelicolor responds to the presence of other actinomycetes. Left panel: S. coelicolor alone, other panels show S. coelicolor on the right and a second species on the left. S. coelicolor colonies exhibit different phenotypes depending on the partner's identity [37].

Figure 2. When to Expect Competition. Ecological conditions leading to high selection for the acquisition or expression of competitive phenotypes include (i) high niche overlap between strains, (ii) if they are well-mixed over a spatial scale that is relevant for interactions and (iii) if cells are at a high density relative to available resources. Whether these conditions are met depends on environmental factors listed in the centre (high or low: darker or lighter shading, arrow pointing up or down, respectively) such as nutrient abundance, its complexity, the rate at which other strains are entering the group from the outside [88], the phylogenetic diversity within the community, whether cells are motile or not, whether their environment is viscous and how often it is disturbed in a way that disperses cells to new locations, reducing phylogenetic and spatial structure. Note that the same factor may have opposing effects in promoting the conditions for or against competition (e.g. viscosity allow cells to form clonal patches to avoid competitors, but also leads to high cell density since it is harder for cells to migrate, which selects for increased competition).

Figure 3. Predicted Long-term Consequences of Competition. We show three strains of bacteria that compete with one another initially (high competition, high diversity and low stability, see top left) and the 
possible outcomes of this competition as proposed in the literature. Under the top three scenarios (A-C), we plot the predicted dynamics in competition, community diversity and ecological stability over time, beginning from high competition and diversity and low stability. The dynamics of competition, diversity and stability in the bottom three scenarios (D-F) are less well understood. Dashed lines represent

\section{Table 1. Competitive Phenotypes in Microbes}

\begin{tabular}{|c|c|c|c|}
\hline $\begin{array}{l}\text { Competitive } \\
\text { phenotype }\end{array}$ & Example of molecule type & Competitive effect & Refs \\
\hline $\begin{array}{l}\text { Digestive enzyme } \\
\text { secretion }\end{array}$ & Proteases & $\begin{array}{l}\text { Digest complex nutrients for } \\
\text { growth }\end{array}$ & {$[16,29]$} \\
\hline Siderophore secretion & Pyoverdin & Bind and scavenge iron for growth & {$[129,130]$} \\
\hline $\begin{array}{l}\text { Production of structural } \\
\text { and motility molecules }\end{array}$ & $\begin{array}{l}\text { Surfactants, rhamnolipids, } \\
\text { EPS, proteins, DNA, } \\
\text { adhesion and anti-adhesion } \\
\text { molecules }\end{array}$ & $\begin{array}{l}\text { Maintain established niche or } \\
\text { colonise a new niche }\end{array}$ & $\begin{array}{l}{[18,20,} \\
22,24,131, \\
132]\end{array}$ \\
\hline Antibiotic production & Bacteriocins, toxins, peptides & $\begin{array}{l}\text { Lysis of competitor via non- } \\
\text { contact dependent chemical } \\
\text { warfare }\end{array}$ & {$[35,38,99]$} \\
\hline $\begin{array}{l}\text { Type VI secretion } \\
\text { systems (T6SS) }\end{array}$ & $\begin{array}{l}\text { Stabbing structures that } \\
\text { release lethal effector } \\
\text { molecules and enzymes }\end{array}$ & $\begin{array}{l}\text { Lysis of competitor via contact } \\
\text { dependent chemical warfare }\end{array}$ & $\begin{array}{l}{[5,34,43,} \\
44]\end{array}$ \\
\hline $\begin{array}{l}\text { Altering metabolic } \\
\text { regulation }\end{array}$ & - & $\begin{array}{l}\text { Better utilisation of substrates in } \\
\text { variable environments }\end{array}$ & $\begin{array}{l}{[8-10,47} \\
133]\end{array}$ \\
\hline $\begin{array}{l}\text { Reduced expression of } \\
\text { costly genes }\end{array}$ & $\begin{array}{l}\text { Secreted molecules that act } \\
\text { as public goods, e.g. } \\
\text { digestive enzymes and } \\
\text { siderophores }\end{array}$ & $\begin{array}{l}\text { Exploit production of higher } \\
\text { producing cells, resulting in } \\
\text { growth advantage }\end{array}$ & $\begin{array}{l}{[13,16,} \\
102,105, \\
130]\end{array}$ \\
\hline $\begin{array}{l}\text { Production of non- } \\
\text { biocidal molecules }\end{array}$ & $\begin{array}{l}\text { Surfactin, anti-adhesion } \\
\text { molecules, nucleases, } \\
\text { proteases }\end{array}$ & $\begin{array}{l}\text { Disperse competitors out of niche, } \\
\text { degrade biofilm matrix }\end{array}$ & $\begin{array}{l}{[27,28} \\
134,135]\end{array}$ \\
\hline Inhibit quorum sensing & $\begin{array}{l}\text { Quorum sensing inhibitors or } \\
\text { quenchers }\end{array}$ & Inhibit cell-to-cell communication & $\begin{array}{l}{[32,33} \\
136]\end{array}$ \\
\hline
\end{tabular}


2 Bren, A., et al. (2013) The last generation of bacterial growth in limiting nutrient. BMC Systems Biology 7, 1

3 Aldén, L., et al. (2001) Rapid method of determining factors limiting bacterial growth in soil. Applied and environmental microbiology 67, 1830-1838

4 Kehl-Fie, T.E. and Skaar, E.P. (2010) Nutritional immunity beyond iron: a role for manganese and zinc. Current opinion in chemical biology 14, 218-224

5 Borgeaud, S., et al. (2015) The type VI secretion system of Vibrio cholerae fosters horizontal gene transfer. Science 347, 63-67

6 Freese, P.D., et al. (2014) Genetic drift suppresses bacterial conjugation in spatially structured populations. Biophysical Journal 106, 944-954

7 Niehus, R., et al. (2015) Migration and horizontal gene transfer divide microbial genomes into multiple niches. Nature Communications 6, 8924

8 MacLean, R.C. and Gudelj, I. (2006) Resource competition and social conflict in experimental populations of yeast. Nature $441,498-501$

9 Pfeiffer, T., et al. (2001) Cooperation and competition in the evolution of ATP-producing pathways. Science 292, 504-507

10 Vulic, M. and Kolter, R. (2001) Evolutionary Cheating in Escherichia coli Stationary Phase Cultures. Genetics 158, 519-526

11 Morris, J.J. (2015) Black Queen evolution: the role of leakiness in structuring microbial communities. Trends in Genetics 31, 475-482

12 Morris, J.J., et al. (2012) The Black Queen Hypothesis: evolution of dependencies through adaptive gene loss. mBio 3, e00036-12

13 Cordero, O.X., et al. (2012) Public good dynamics drive evolution of iron acquisition strategies in natural bacterioplankton populations. Proceedings of the National Academy of Sciences 109, 20059-20064 14 Ghoul, M., et al. (2014) An experimental test of whether cheating is context dependent. Journal of Evolutionary Biology 27, 551-556

15 Kümmerli, R., et al. (2009) Viscous medium promotes cooperation in the pathogenic bacterium Pseudomonas aeruginosa. Proc R Soc Lond Biol 276, 3531-3538

16 Diggle, S.P., et al. (2007) Cooperation and conflict in quorum-sensing bacterial populations. Nature 450, 411-U417

17 Schuster, M., et al. (2013) Acyl-homoserine lactone quorum sensing: from evolution to application. Annual Review of Microbiology 67, 43-63

$18 \mathrm{Kim}, \mathrm{W}$., et al. (2014) Importance of positioning for microbial evolution. Proceedings of the National Academy of Sciences of the United States of America 111, E1639-1647

19 Bucci, V., et al. (2011) The evolution of bacteriocin production in bacterial biofilms. The American naturalist 178, E162-173

20 An, D., et al. (2006) Quorum sensing and motility mediate interactions between

Pseudomonas aeruginosa and Agrobacterium tumefaciens in biofilm cocultures. Proceedings of the National Academy of Sciences of the United States of America 103, 3828-3833

$21 \mathrm{Kim}, \mathrm{W}$., et al. (2016) Rapid radiation in bacteria leads to a division of labour. Nature Communications 7, 10508

22 Schluter, J., et al. (2015) Adhesion as a weapon in microbial competition. The ISME journal $9,139-149$

23 Xavier, J.B. and Foster, K.R. (2007) Cooperation and conflict in microbial biofilms. PNAS $104,876-881$

24 Nadell, C.D. and Bassler, B.L. (2011) A fitness trade-off between local competition and dispersal in Vibrio cholerae biofilms. PNAS 108, 14181-14185

25 Hibbing, M.E., et al. (2010) Bacterial competition: Surviving and thriving in the microbial jungle. Nature Reviews Microbiology 8, 15-25 
526

527

528

529

530

531

532

533

534

535

536

537

538

539

540

541

542

543

544

545

546

547

548

549

550

551

552

553

554

555

556

557

558

559

560

561

562

563

564

565

566

567

568

569

570

571

572

573

574

575

576

26 Stacy, A., et al. (2016) The biogeography of polymicrobial infection. Nature Reviews Microbiology 14, 93-105

27 Rendueles, O., et al. (2011) Screening of Escherichia coli species biodiversity reveals new biofilm-associated antiadhesion polysaccharides. mBio 2, e00043-00011

28 Valle, J., et al. (2006) Broad-spectrum biofilm inhibition by a secreted bacterial polysaccharide. Proceedings of the National Academy of Sciences of the United States of America 103, 12558-12563

29 Rendueles, O. and Ghigo, J.-M. (2012) Multi-species biofilms: how to avoid unfriendly neighbors. FEMS microbiology reviews 36, 972-989

30 Anderson, G. and O'Toole, G. (2008) Innate and induced resistance mechanisms of bacterial biofilms. Current Topics in Microbiology \& Immunology 322, 85-105

31 Augustine, N., et al. (2010) Inhibition of Vibrio cholerae biofilm by AiiA enzyme produced from Bacillus spp. Archives of Microbiology 192, 1019-1022

32 Musthafa, K.S., et al. (2011) Antipathogenic potential of marine Bacillus sp. SS4 on N-acylhomoserine-lactone-mediated virulence factors production in Pseudomonas aeruginosa (PAO1). J Biosciences 36, 55-67

33 Christiaen, S., et al. (2011) Isolation and identification of quorum quenching bacteria from environmental samples. Journal of Microbiology Methods 87, 213-219

34 Basler, M., et al. (2013) Tit-for-Tat: Type VI Secretion System Counterattack during Bacterial Cell-Cell Interactions. Cell 152, 884-894

35 Chao, L. and Levin, B.R. (1981) Structured habitats and the evolution of anticompetitor toxins in bacteria. Proceedings of the National Academy of Sciences of the United States of America 78, 6324-6328

36 Mitri, S., et al. (2015) Resource limitation drives spatial organization in microbial groups. The ISME journal 10, 1471-1482

37 Traxler, M.F., et al. (2013) Interspecies interactions stimulate diversification of the

Streptomyces coelicolor secreted metabolome. mBio 4, e00459-13

38 Riley, M.A. and Gordon, D.M. (1999) The ecological role of bacteriocins in bacterial

competition. Trends Microbiol 7, 129-133

39 Davies, J., et al. (2006) The world of subinhibitory antibiotic concentrations. Current opinion in microbiology 9, 445-453

40 Romero, D., et al. (2011) Antibiotics as signal molecules. Chemical reviews 111, 5492-5505 41 Abrudan, M.l., et al. (2015) Socially mediated induction and suppression of antibiosis during bacterial coexistence. Proceedings of the National Academy of Sciences of the United States of America 112, 11054-11059

42 Cornforth, D.M. and Foster, K.R. (2015) Antibiotics and the art of bacterial war. Proceedings of the National Academy of Sciences of the United States of America 112, 10827-10828

43 Russell, A.B., et al. (2014) Type VI secretion system effectors: poisons with a purpose. Nature Reviews Microbiology 12, 137-148

44 Macintyre, D.L., et al. (2010) The Vibrio cholerae type VI secretion system displays antimicrobial properties. PNAS 107, 19520-19524

45 Shapiro, B.J., et al. (2012) Population genomics of early events in the ecological differentiation of bacteria. Science 336, 48-51

46 Takeuchi, N., et al. (2015) Gene-specific selective sweeps in bacteria and archaea caused by negative frequency-dependent selection. BMC Biology 13, 20

47 Ackermann, M. (2015) A functional perspective on phenotypic heterogeneity in microorganisms. Nature Reviews Microbiology 13, 497-508

48 Stewart, P.S. and Franklin, M.J. (2008) Physiological heterogeneity in biofilms. Nature

reviews. Microbiology 6, 199-210

49 Chai, Y.R., et al. (2008) Bistability and biofilm formation in Bacillus subtilis. Mol Microbiol 67, 254-263 
577

578

579

580

581

582

583

584

585

586

587

588

589

590

591

592

593

594

595

596

597

598

599

600

601

602

603

604

605

606

607

608

609

610

611

612

613

614

615

616

617

618

619

620

621

622

623

624

625

626

50 Schreiber, F., et al. (2016) Phenotypic heterogeneity driven by nutrient limitation promotes growth in fluctuating environments. Nature Microbiology 1, 16055

51 Flores, E. and Herrero, A. (2010) Compartmentalized function through cell differentiation in filamentous cyanobacteria. Nature Reviews Microbiology 8, 39-50

52 Diard, M., et al. (2013) Stabilization of cooperative virulence by the expression of an avirulent phenotype. Nature 494, 353-356

53 Boyer, F., et al. (2009) Dissecting the bacterial type VI secretion system by a genome wide in silico analysis: what can be learned from available microbial genomic resources? BMC

genomics 10, 104

54 Nett, M., et al. (2009) Genomic basis for natural product biosynthetic diversity in the actinomycetes. Natural Product Reports 26, 1362-1384

55 Freilich, S., et al. (2011) Competitive and cooperative metabolic interactions in bacterial communities. Nature Communications 2, 589

56 O'Brien, E., et al. (2015) Using genome-scale models to predict biological capabilities. Cell 161, 971-987

57 Lawrence, D., et al. (2012) Species Interactions Alter Evolutionary Responses to a Novel

Environment. PLoS Biology 10, e1001330

58 Rivett, D.W., et al. (2016) Resource-dependent attenuation of species interactions during

bacterial succession. The ISME journal doi: 10.1038/ismej.2016.11

59 Fiegna, F., et al. (2015) Evolution of species interactions determines microbial community productivity in new environments. The ISME Journal 9, 1235-1245

60 Kinkel, L.L., et al. (2014) Sympatric inhibition and niche differentiation suggest alternative coevolutionary trajectories among Streptomycetes. The ISME journal 8, 249-256

61 Schulz-Bohm, K., et al. (2015) A fragrant neighborhood: volatile mediated bacterial interactions in soil. Frontiers in Microbiology 6, 1-11

62 Vetsigian, K., et al. (2011) Structure and evolution of Streptomyces interaction networks in soil and in silico. PLoS Biology 9, e1001184

63 Stein, R.R., et al. (2013) Ecological modeling from time-series inference: insight into dynamics and stability of intestinal microbiota. PLoS computational biology 9, e1003388 64 Coyte, K.Z., et al. (2015) The ecology of the microbiome: Networks, competition, and stability. Science 350, 663-666

65 Levy, R. and Borenstein, E. (2013) Metabolic modeling of species interaction in the human microbiome elucidates community-level assembly rules. Proceedings of the National Academy of Sciences of the United States of America 110, 12804-12809

66 Bik, E.M., et al. (2010) Bacterial diversity in the oral cavity of 10 healthy individuals. The ISME journal 4, 962-974

67 Smillie, C.S., et al. (2011) Ecology drives a global network of gene exchange connecting the human microbiome. Nature 480, 241-244

68 Harcombe, W. (2010) Novel cooperation experimentally evolved between species. Evolution 64, 2166-2172

69 Shou, W., et al. (2007) Synthetic cooperation in engineered yeast populations. Proceedings of the National Academy of Sciences of the United States of America 104, 1877-1882

70 Pande, S., et al. (2015) Metabolic cross-feeding via intercellular nanotubes among bacteria. Nature Communications 6, 6238

71 Hansen, S.K., et al. (2007) Evolution of species interactions in a biofilm community. Nature 445, 533-536

72 Fischbach, M.A. and Walsh, C.T. (2009) Antibiotics for Emerging Pathogens. Science 325, 1089-1093

73 Livermore, D.M. and Antimicrobial, B.S. (2011) Discovery research: the scientific challenge of finding new antibiotics. J Antimicrob Chemoth 66, 1941-1944 
627

628

629

630

631

632

633

634

635

636

637

638

639

640

641

642

643

644

645

646

647

648

649

650

651

652

653

654

655

656

657

658

659

660

661

662

663

664

665

666

667

668

669

670

671

672

673

674

675

676

677

74 van Gestel, J., et al. (2014) Density of founder cells affects spatial pattern formation and cooperation in Bacillus subtilis biofilms. The ISME journal 8, 2069-2079

75 Lloyd, D.P. and Allen, R.J. (2015) Competition for space during bacterial colonization of a surface. Journal of the Royal Society Interface 12, 20150608

76 Bellucci, M., et al. (2015) A preliminary and qualitative study of resource ratio theory to nitrifying lab-scale bioreactors. Microbial Biotechnology 8, 590-603

77 Miller, T.E., et al. (2005) A critical review of twenty years' use of the Resource-Ratio Theory. The American Naturalist 165, 439-448

78 Zelezniak, A., et al. (2015) Metabolic dependencies drive species co-occurrence in diverse microbial communities. Proceedings of the National Academy of Sciences 112, 201421834 79 Hardin, G. (1960) The competitive exclusion principle. Science 131, 1292-1297

80 Mitri, S. and Foster, K.R. (2013) The Genotypic View of Social Interactions in Microbial Communities. Annual Review of Genetics 47, 247-273

81 Nadell, C.D., et al. (2010) Emergence of spatial structure in cell groups and the evolution of cooperation. PLoS Computational Biology 6, e1000716

82 Kinkel, L.L., et al. (2011) A coevolutionary framework for managing disease-suppressive soils. Annual Reviews of Phytopathology 49, 47-67

83 Cardinale, B.J. (2011) Biodiversity improves water quality through niche partitioning. Nature 472, 86-89

84 Momeni, B., et al. (2013) Spatial self-organization favors heterotypic cooperation over cheating. eLife 2, 1-18

85 Mitri, S., et al. (2011) Social evolution in multispecies biofilms. PNAS 108, 10839-10846 86 Muller, M.J.I., et al. (2014) Genetic drift opposes mutualism during spatial population expansion. Proceedings of the National Academy of Sciences 111, 1037-1042

87 Estrela, S. and Brown, S.P. (2013) Metabolic and Demographic Feedbacks Shape the Emergent Spatial Structure and Function of Microbial Communities. PLoS Computational Biology 9, e1003398

88 Kerr, B., et al. (2006) Local migration promotes competitive restraint in a host-pathogen 'tragedy of the commons'. Nature $442,75-78$

89 Cornforth, D.M. and Foster, K.R. (2013) Competition sensing: the social side of bacterial stress responses. Nature Reviews Microbiology 11, 285-293

90 LeRoux, M., et al. (2015) Bacterial danger sensing. Journal of molecular biology 427, 37443753

91 Oliveira, N.M., et al. (2015) Biofilm formation as a response to ecological competition. PLoS Biology 13, e1002232

92 LeRoux, M., et al. (2015) Kin cell lysis is a danger signal that activates antibacterial pathways of Pseudomonas aeruginosa. eLife 4, e05701

93 Rosenberg, G., et al. (2016) Not so simple, not so subtle: the interspecies competition between Bacillus simplex and Bacillus subtilis and its impact on the evolution of biofilms. $n p j$ Biofilms and Microbiomes 2, 15027

94 Kelsic, E.D., et al. (2015) Counteraction of antibiotic production and degradation stabilizes microbial communities. Nature 521, 516-519

95 Allesina, S. and Levine, J.M. (2011) A competitive network theory of species diversity. Proceedings of the National Academy of Sciences of the United States of America 108, 56385642

96 Louca, S. and Doebeli, M. (2015) Transient dynamics of competitive exclusion in microbial communities. Environmental microbiology 18, 1863-1874

97 Hallatschek, O., et al. (2007) Genetic drift at expanding frontiers promotes gene segregation. PNAS 104, 19926-19930

98 Korolev, K.S., et al. (2012) Selective sweeps in growing microbial colonies. Physical biology 9,026008 
678

679

680

681

682

683

684

685

686

687

688

689

690

691

692

693

694

695

696

697

698

699

700

701

702

703

704

705

706

707

708

709

710

711

712

713

714

715

716

717

718

719

720

721

722

723

724

725

726

727

728

99 Kerr, B., et al. (2002) Local dispersal promotes biodiversity in a real-life game of rock-paperscissors. Nature 418, 171-174

100 Rainey, P.B. and Travisano, M. (1998) Adaptive radiation in a heterogeneous environment. Nature 394, 69-72

101 Estrela, S., et al. (2015) Private benefits and metabolic conflicts shape the emergence of microbial interdependencies. Environmental microbiology 18, 1415-1427

102 Gore, J., et al. (2009) Snowdrift game dynamics and facultative cheating in yeast. Nature 459, 253-256

103 Morris, J.J., et al. (2014) Coexistence of evolving bacteria stabilized by a shared Black Queen function. Evolution 68, 2960-2971

104 Sanchez, A. and Gore, J. (2013) Feedback between Population and Evolutionary Dynamics Determines the Fate of Social Microbial Populations. PLoS Biology 11, e1001547

105 Diard, M., et al. (2013) Stabilization of cooperative virulence by the expression of an avirulent phenotype. Nature 494, 353-358

106 Narisawa, N., et al. (2008) Coexistence of antibiotic-producing and antibiotic-sensitive bacteria in biofilms is mediated by resistant bacteria. Applied and environmental microbiology 74, 3887-3894

107 Czárán, T.L., et al. (2002) Chemical warfare between microbes promotes biodiversity. PNAS 99, 786-790

108 Sachs, J.L. (2012) The origins of cooperative bacterial communities. mBio 3, e00099-00012 109 Biernaskie, J., et al. (2013) Multicoloured greenbeards, bacteriocin diversity and the rockpaper-scissors game. Journal of Evolutionary Biology 26, 2081-2094

110 Borenstein, D.B., et al. (2015) Established Microbial Colonies Can Survive Type VI

Secretion Assault. PLoS computational biology 11, e1004520

111 Schlatter, D.C. and Kinkel, L.L. (2015) Do tradeoffs structure antibiotic inhibition, resistance, and resource use among soil-borne Streptomyces? BMC evolutionary biology 15, 186 112 Conlin, P.L., et al. (2014) Games of life and death: antibiotic resistance and production through the lens of evolutionary game theory. Current opinion in microbiology 21, 35-44 113 Celiker, H. and Gore, J. (2012) Competition between species can stabilize public-goods cooperation within a species. Molecular systems biology 8, 621

114 Thompson, J.N. (2005) The Geographic Mosaic of Coevolution. The University of Chicago Press

115 Fredrickson, J.K. (2015) Ecological communities by design. Science 348, 1425-1427

116 Faust, K., et al. (2015) Cross-biome comparison of microbial association networks.

Frontiers in Microbiology 6, 1200

117 Lima-Mendez, G., et al. (2015) Determinants of community structure in the global plankton interactome. Science 348, 1262073

118 Berry, D. and Widder, S. (2014) Deciphering microbial interactions and detecting keystone species with co-occurrence networks. Frontiers in Microbiology 5, 1-14

119 Embree, M., et al. (2015) Networks of energetic and metabolic interactions define dynamics in microbial communities. PNAS 112, 15450-15455

120 Güell, M., et al. (2011) Bacterial transcriptomics: what is beyond the RNA horiz-ome?

Nature Reviews Microbiology 9, 658-669

121 Patti, G.J., et al. (2012) Innovation: Metabolimics: the apogee of the omics trilogy. Nature Reviews Molecular Cell Biology 13, 263-269

122 Ernebjerg, M. and Kishony, R. (2012) Distinct growth strategies of soil bacteria as revealed by large-scale colony tracking. Applied and Environmental Microbiology 78, 1345-1352

123 Mounier, J., et al. (2008) Microbial Interactions within a Cheese Microbial Community.

Applied and Environmental Microbiology 74, 172-181

124 Wessel, A.K., et al. (2013) Going local: technologies for exploring bacterial

microenvironments. Nature Reviews Microbiology 11, 337-348 
125 Harcombe, William R., et al. (2014) Metabolic Resource Allocation in Individual Microbes Determines Ecosystem Interactions and Spatial Dynamics. Cell Reports 7, 1104-1115 126 Klitgord, N. and Segrè, D. (2010) Environments that induce synthetic microbial ecosystems. PLoS computational biology 6, e1001002

733127 Gardner, A., et al. (2004) Bacteriocins, spite and virulence. Proceedings. Biological

734 sciences / The Royal Society 271, 1529-1535

735128 Maynard Smith, J. (1982) Evolution and the Theory of Games.

736129 Scholz, R.L. and Greenberg, E.P. (2015) Sociality in Escherichia coli: Enterochelin Is a 737 Private Good at Low Cell Density and Can Be Shared at High Cell Density. J Bacteriol 197, $738 \quad 2122-2128$

739130 Griffin, A.S., et al. (2004) Cooperation and competition in pathogenic bacteria. Nature 430, $740 \quad 1024-1027$

741131 Romero, D., et al. (2011) An accessory protein required for anchoring and assembly of 742 amyloid fibres in B. subtilis biofilms. Mol Microbiol 80, 1155-1168

743132 Whitchurch, C.B., et al. (2002) Extracellular DNA required for bacterial biofilm formation. 744 Science 295, 1487-1487

745133 Kotte, O., et al. (2014) Phenotypic bistability in Escherichia colis central carbon

746 metabolism. Molecular Systems Biology 10, 736

747134 Mowat, E., et al. (2010) Pseudomonas aeruginosa and their small diffusible extracellular 748 molecules inhibit Aspergillus fumigatus biofilm formation. Fems Microbiol Lett 313, 96-102

749135 Jiang, P., et al. (2011) Antibiofilm activity of an exopolysaccharide from marine bacterium

$750 \quad$ Vibrio sp. QY101. PloS one 6, e18514

751136 Dong, Y.H., et al. (2002) Identification of quorum-quenching $\mathrm{N}$-acyl homoserine lactonases

752 from Bacillus species. Applied and Environmental Microbiology 68, 1754-1759 


\section{Outstanding Questions}

- What is the effect of DNA uptake on fitness?

- How does the environment dictate the prevalence of competition?

- What determines the ability of a strain to resist invasion?

- Is competition always a temporary state or do constant battlefields exist? How stable are different outcomes (Figure 3D-F)?

- Is it possible to manipulate competition by altering environmental conditions?

- How aggressive are secondary metabolites commonly found in genomic data?

- How variable is the expression of competitive phenotypes within a population of clonal cells, and how does this heterogeneity affect the success of genotypes? 


\section{Figure 1}

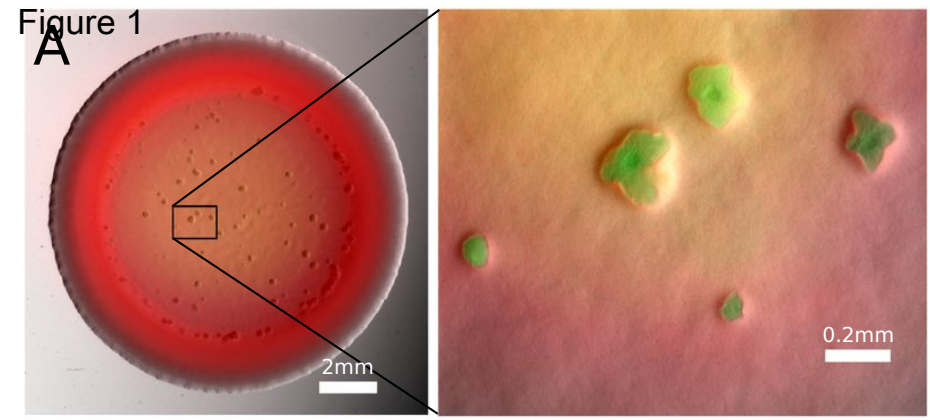

B

$0.2 \mathrm{~mm}$
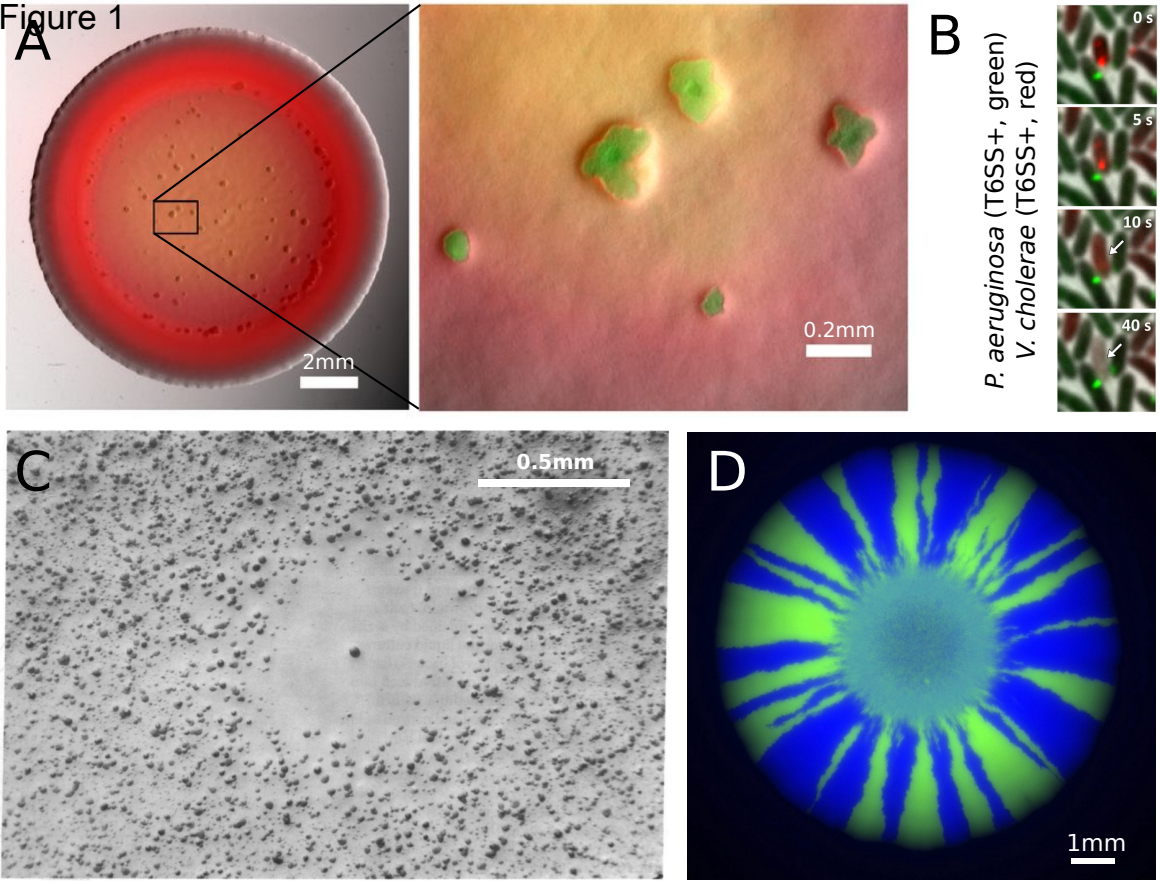

E ()
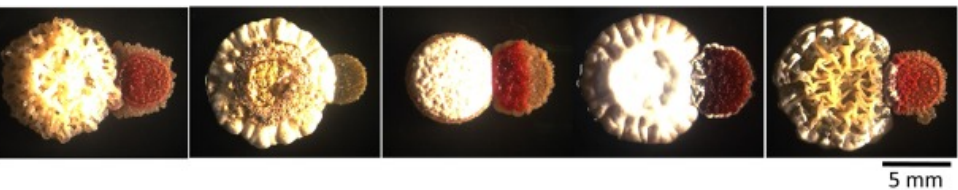
Fiqure 2 high selection for competition

\section{Low selection for competition}

Low metabolic overlap

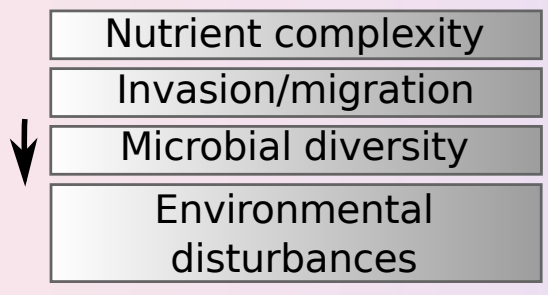

Nutrient concentration

$\uparrow$

Cell motility

Environmental disturbances

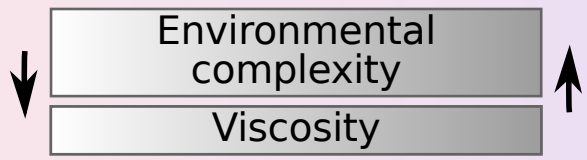

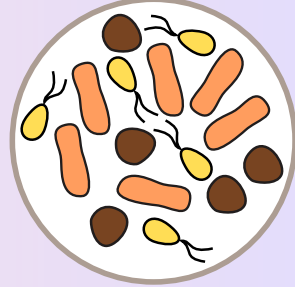

Structured

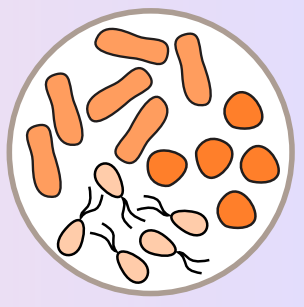

High cell density

Low cell density

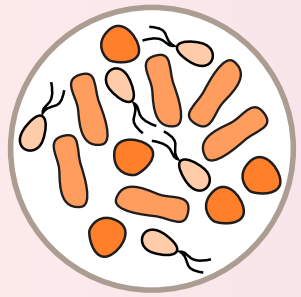

$\uparrow \frac{\text { Nutrient concentration }}{\mid \text { Viscosity }} \downarrow$

$\downarrow \square$ Cell motility

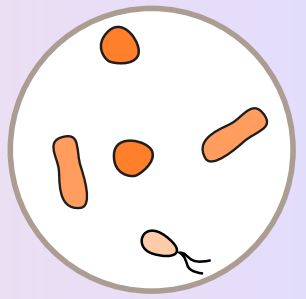




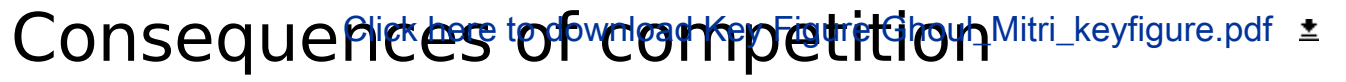

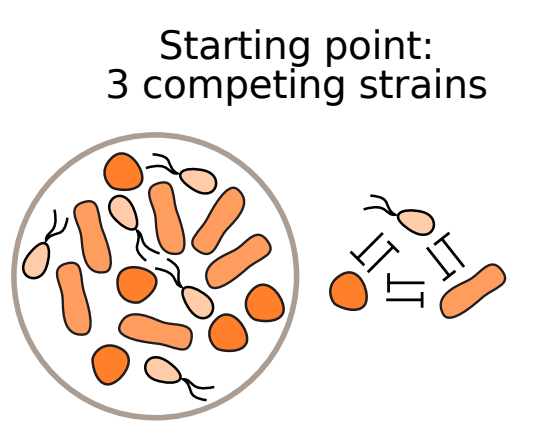

خo Species 1

Species 2

Species 3

$\rightarrow$ Inhibition

$\rightarrow$ Promotion

Antibiotic produced

3 by 1

Antibiotic produced by 1 , suppressed by 2

Lysed cell

Predicted dynamics

- Competition

- Diversity

- Ecological stability

- - Educated guess, to be tested
A Competitive exclusion
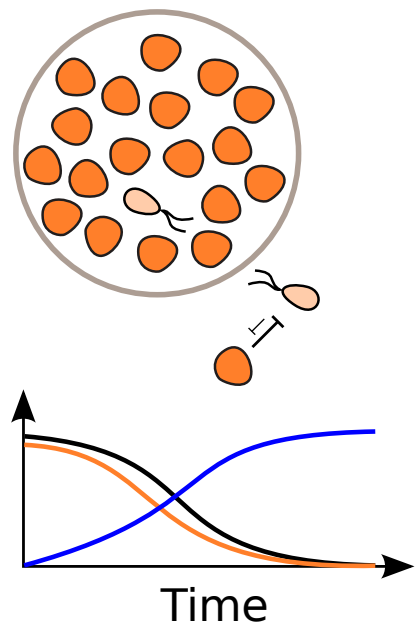

D Exploitation of secretions
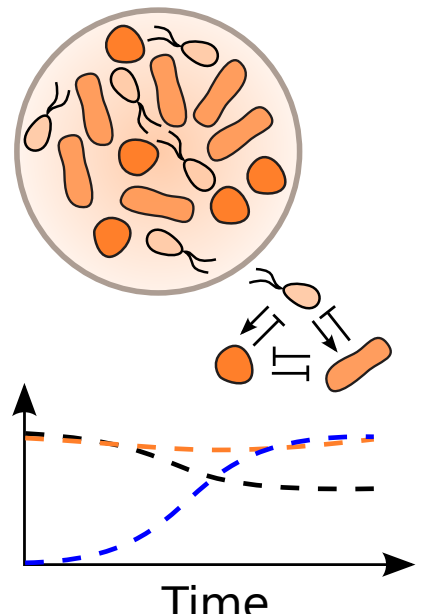

B Niche partitioning (resources)

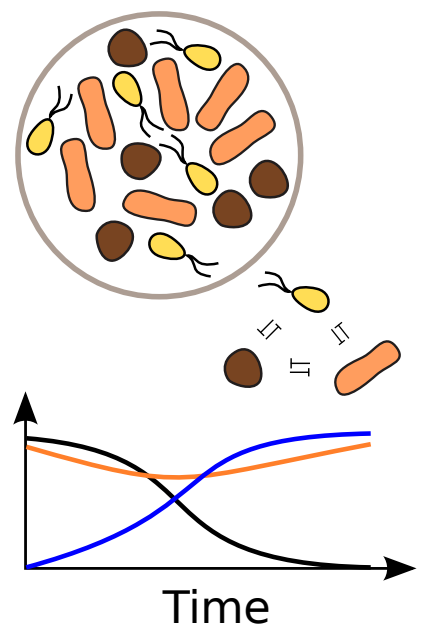

E $\begin{gathered}\text { Continued } \\ \text { aggression }\end{gathered}$
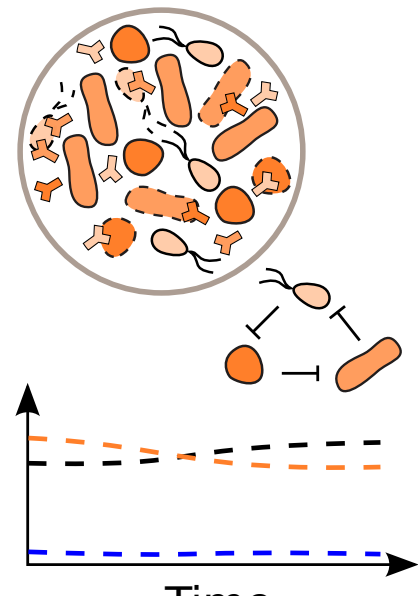

C Niche partitioning (space)

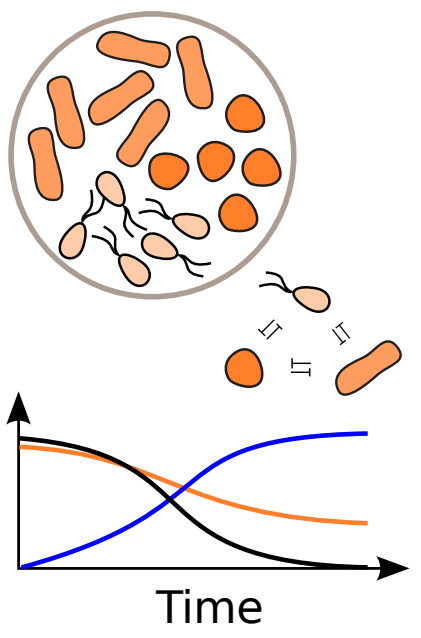

F Counteracting
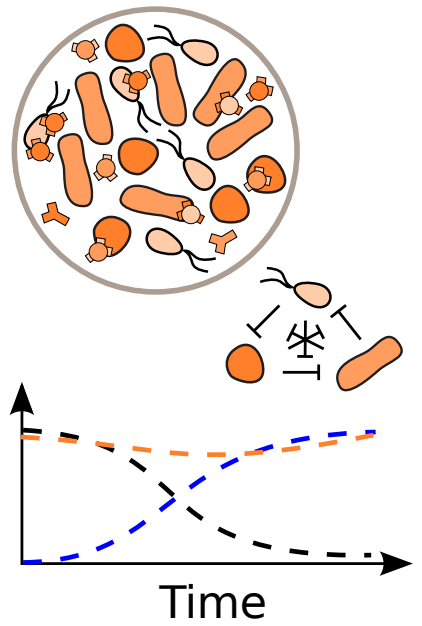